\title{
Effects of Light With Reduced Short Wavelength Components on Parameters of Circadian Rhythm and Performance in an Experimental Night Shift Model
}

\author{
Georg Hoffmann*,1, Veronika Leichtfried ${ }^{1}$, Andrea Griesmacher ${ }^{2}$, Christian Bartenbach ${ }^{3}$, Markus \\ Canazei $^{3}$, Siegmund Staggl ${ }^{3}$ and Wolfgang Schobersberger ${ }^{1}$
}

\author{
${ }^{I}$ Institute for Leisure, Travel, and Alpine Medicine; University for Health Sciences, Medical Informatics, and Technol- \\ ogy, Eduard Wallnoefer-Zentrum 1, A-6060 Hall, Austria \\ ${ }^{2}$ Central Institute for Medical and Chemical Laboratory Diagnostics, University Hospital Innsbruck-TILAK, Innsbruck, \\ Austria \\ ${ }^{3}$ Bartenbach LichtLabor, Rinner Strasse 14, 6071 Aldrans, Austria
}

\begin{abstract}
Shift work is associated with alterations in physiological circadian patterns resulting in chronic diseases, e.g. cardiovascular disorders or major depression. The intensity and spectral composition of light is known to affect the $24 \mathrm{~h}-$ rhythm of our body. We investigated the effects of two different lighting environments on parameters of circadian rhythm and performance in healthy volunteers during an experimental night shift. Test light with a low color temperature was compared to normal light with a higher color temperature. Melatonin synthesis, red and white blood count, blood pressure, heart rate and indicators of performance were analyzed. Nocturnal increases in melatonin were more pronounced under low color temperature lighting conditions. This was not associated with limited degrees of arousal or vigilance. Maintenance of a normal nocturnal rhythm of melatonin with adapted illumination may provide a benefit for employees wellbeing without affecting their productivity.
\end{abstract}

\section{INTRODUCTION}

An increasing number of employees in industrialized nations perform night shift work on a regular basis. Among the risks associated with night shift jobs are increased error and accident rates as well as production of substandard goods $[1,2]$. While the deliberate disturbance of a physiological circadian rhythm is supposed to be involved in the pathogenesis of cardiovascular disorders or major depression $[3,4]$, the evidence connecting shift work and an increased incidence of certain types of tumors, e.g. colorectal and breast cancer, is discussed controversially [5-7]. Although the exact mechanisms underlying these detrimental processes are unclear at present, nocturnal bright light exposure is regarded to be one decisive factor in the development of these harmful events. Circadian rhythms can be found in every cell of our body. The cellular oscillations are coordinated via superior centres in the suprachiasmatic nucleus (SCN) representing the master clock of the human organism. The activities of the SCN are synchronized by exogenous factors appearing in a periodical fashion with the natural light-dark cycle being the most prominent Zeitgeber. Light signals are most likely received by melanopsin-containing ganglion cells already described in the retina of rodents [8], primates [9], and humans [10]. The mode of action and the sensitivity of these cells were primarily investigated in connection with

*Address correspondence to this author at the Institute for Leisure, Travel, and Alpine Medicine; University for Health Sciences, Medical Informatics, and Technology, Eduard Wallnoefer-Zentrum 1, A-6060 Hall, Austria; E-mail: Georg.Hoffmann@umit.at the light-induced suppression of melatonin synthesis. Melatonin is a hormone produced in the pineal gland. Synthesis and release of melatonin into the blood stream oscillates in a circadian pattern with the highest concentrations found late at night (at 04:00 a.m.) and decreasing values in the course of the day reaching a nadir at $\sim 04: 00$ p.m. [11]. Inhibition of melatonin release triggered by light was first described by Klein and Weller [12] in rodents while Lewy et al. [13] detected a corresponding effect of light on melatonin in humans. A light intensity of 100 lux seems to be efficient to attenuate pineal synthesis of the hormone [14]. Besides its intensity, the spectral composition of light exerts a substantial impact on melatonin generation. The action spectrum of melanopsin-containing ganglion cells exhibit a maximal photosensitivity at $\sim 460 \mathrm{~nm}[15,16]$. Therefore, the application of filtered light with reduced short wavelength components (i.e. low color temperature) represents a potential concept for the mitigation or avoidance of light-induced detrimental consequences during night shift. The aim of the present study was to investigate the effects of two differently illuminated office workplaces on melatonin synthesis in an experimental night shift setup. Light with a low color temperature $(1700 \mathrm{~K}$ at eye level) was compared to light with a higher color temperature $(6300 \mathrm{~K}$ at eye level). In addition to melatonin, other physiological markers following a circadian pattern (heart rate, blood pressure, white and red blood count) were investigated. Ergonomical interventions aiming at the maintenance of a biological circadian profile can only be successful on the condition that they do not interfere with the employees productivity. Thus, a number of tests were performed to elucidate the effects of the different 
lighting environments on the efficiency of the study participants.

\section{MATERIALS AND METHODOLOGY}

\section{Study Participants and Study Design}

11 healthy male volunteers (age: 23 (range: 22-29) years; body weight: 74 (range: 62-84) kg; height: 177 (range: 172$181) \mathrm{cm}$ ) without any known metabolic, cardiovascular, renal, hepatic, neurological, psychiatric or sleeping disorders requiring acute or chronic medication participated in our study. Test persons had not undertaken shift work or transmeridian travel in the month prior to the study. Subjects were instructed to refrain from excessive physical exercise, alcohol consumption, and over-the-counter medication during the course of the study. At the beginning of every night of the study, a sleeping protocol of the preceding night was recorded. No exceptional sleeping behaviour was reported. The project was approved by the Ethics Committee of the Medical University of Innsbruck, and all participants gave written informed consent. The study was performed at the Bartenbach LichtLabor, Aldrans, Austria following a crossover design with three consecutive days of experimental office work in two different lighting environments but otherwise constant workplace conditions (area: $20 \mathrm{~m}^{2}$; ceiling height: $2.6 \mathrm{~m}$; temperature: $23^{\circ} \mathrm{C} \pm 2^{\circ} \mathrm{C}$ regulated via air handling unit, environmental luminance: $300 \mathrm{~cd} / \mathrm{m}^{2}$ ). The volunteers were randomly assigned to start with three consecutive nights of simulated shift work either in an unfiltered bright light environment (luminaire: wallwasher, color temperature $6300 \mathrm{~K}$ at eye level; color rendering index: 89) or in a lighting environment with reduced short-wavelength components (luminaire: wallwasher, color temperature $1700 \mathrm{~K}$ at eye level; color rendering index: 80). Following an 11-day break, the study participants conducted a second run of three consecutive night shifts in the other lighting environment. Each night shift started at 10:00 p.m. at night and ended at 06:00 a.m. in the morning.

\section{Laboratory Measurements}

At the beginning (22:00 p.m., labeled B1 throughout the manuscript) and at the end (06:00 a.m., labeled B2 throughout the manuscript) of each night of the study, blood samples were taken from an antecubital vein and processed at the Central Institute for Medical and Chemical Laboratory Diagnostics, Innsbruck, Austria. Erythrocyte count, hemoglobin, hematocrit, and white blood cell count were measured by standard methods.

Serum melatonin was assayed using a commercially available radio-immunoassay kit (Buehlmann Melatonin direct RIA, Buehlmann Laboratories AG, Schoenenbuch, Switzerland) with an analytical sensitivity of $0.84 \mathrm{pg}$ melatonin $/ \mathrm{ml}$. In brief, samples, assay controls, and melatonin in human serum matrix as calibrators were incubated with ${ }^{125} \mathrm{I}-$ labeled melatonin and a specific rabbit anti-melatonin antibody for $20 \mathrm{~h}$ at $4^{\circ} \mathrm{C}$ in polystyrene tubes. Subsequently, solid phase bound anti-rabbit second antibody was added into each tube and incubated for an additional $15 \mathrm{~min}$ at $4^{\circ} \mathrm{C}$ to precipitate the antibody-bound fraction. Finally, ultrapure water was added and the tubes were centrifuged at $2000 \mathrm{~g}$ for 2 min at room temperature. Supernatants were discarded and ${ }^{125}$ I activity in the precipitates was detected for $2 \mathrm{~min}$ in a $\gamma$ - counter. Unknown samples were calculated using a standard curve generated by the calibrators provided by the manufacturer. All measurements were performed in duplicate. Data are given as pg melatonin/ml. All melatonin analyses were done at the Central Institute for Medical and Chemical Laboratory Diagnostics, Innsbruck, Austria.

\section{Blood Pressure and Heart Rate}

Blood pressure and heart rate were recorded using an automatic device (Omron M5, Omron Medizintechnik Handelsgesellschaft $\mathrm{mbH}$, Mannheim, Germany). First data were collected at the beginning of the experimental night shift. Subsequently, a number of six measurements were performed between the ability testings adding up to a total of seven recordings per night labeled M1 to M7. Approximate time points for each measurement were: M1 at 10:30 p.m.; M2 at 11:30 p.m.; M3 at 0:15 a.m.; M4 at 1:45 a.m.; M5 at 2:45 a.m.; M6 at 3:30 a.m.; and M7 at 5:30 a.m., respectively. Mean values of three readings were used for statistical analyses.

\section{Ability Test Systems}

Simulated office work consisted of general and special ability tests all being part of the Vienna Test System ${ }^{\circledR}$ (Dr. G. Schuhfried Ltd., Moedling, Austria). The tests were performed twice each night with the first test being labeled A1 and the second being labeled A2 throughout the manuscript.

\section{Flicker/Fusion Frequency}

In order to evaluate central nervous activation or arousal, flicker/fusion frequency analyses were performed at 10:15 p.m. and at 5:30 a.m. The test is divided into two parts. At first, frequency of flickering light is increased until a constant light is perceived ("fusion frequency"). In the second part of the experiment, the frequency of constant light is decreased until the subject senses flickering light again ("flicker frequency"). Both transitions are captured by the study participants via pressing a key at the flicker/fusion device. The critical frequency is stored and threshold values are calculated from eight consecutive measurements. Total running time of this test was $10 \mathrm{~min}$. Abbreviations for evaluation scores used in the manuscript are FUF for "fusion frequency" and FLF for "flicker frequency", respectively.

\section{Continuous Attention}

Long-term selective attention and concentration ability were assessed twice per night by the continuous attention test at 11:00 p.m. and at 3:00 a.m. Rows of triangles were presented on a computer monitor with their tips pointing either up or down. A fixed number of tips pointing down had to be responded by the study participants by pushing a reaction button on the keyboard. Duration of the test was $35 \mathrm{~min}$. For analyses, the sum of correct hits (SUMC) was calculated. Auxiliary parameters were the sum of incorrect hits (SUMI), the mean time for correct hits (MEANC), and the mean time for incorrect hits (MEANI).

\section{Reaction Time Analyses}

Potential slow-downs of cognitive speed were assessed by monitoring the three stages of activity regulation (discriminative perception, cognitive processing, and motor response organization) at 11:30 p.m. and at 3:00 a.m., respec- 
tively. Discrimination of figures (circle, rectangle, square, star, cross, ellipse) on a computer monitor was aggravated by partial covering with a grid. Variation of the cognitive processing stage was realized by the search for one or two of two simultaneously presented figures. Variation of motor response organization was achieved by modifying the complexity of the reaction to be executed (either with one finger or with a sequence of three keys). Subjects were guided through the experiments interactively. 14 series with either 20 individual stimuli, each for choice reaction tasks or 16 individual stimuli for visual search tasks, were processed. A total running time of $25 \mathrm{~min}$ was required. Scores are expressed as information processing (IP), perception (PER), and motor response organization (MRO). Additional scoring parameters were choice reaction time (CRT) and reaction time for visual search (VSRT).

\section{Vigilance Testing}

Subjects were required to maintain continuous attention although the working environment became stale and signals appeared only randomly and did not cause agitation. To detect continuous vigilance, stimuli with a low intensity and a low frequency of critical events were provided. A flashing dot on the computer monitor travelling in a circular pattern in distinct intervals had to be observed. At random, the interval was doubled, which had to be recorded by the study participants via pressing a button on the keyboard. Vigilance testing was performed at midnight and at 3:30 a.m. Total running time of the test was $70 \mathrm{~min}$. Main scoring parameter for analyses was the number of correct hits (NUMC), auxiliary values included number of incorrect hits (NUMI), and mean value of reaction time for correct hits (MRTC).

\section{Statistical Analyses}

To compare between three or more matched groups with repeated measures Friedman tests were performed. To exactly compare two paired groups, we used the Wilcoxon matched pairs test. To test whether two variables varied together, a nonparametric Spearman correlation was calculated. For presentation of descriptive data, box and whiskers plots were used. The box represents the median together with the $25^{\text {th }}$ and $75^{\text {th }}$ quartile, while the whiskers show the highest and lowest values, respectively. P-values $<0.05$ were considered to be significant.

\section{RESULTS}

Both lighting environments were tolerated by the study participants without adverse effects and the experiments were completed by all volunteers. Fig. (1) summarizes the results of melatonin measurements performed in serum samples obtained at the beginning (B1) and at the end (B2) of each night of the study either in the room with filtered test light (room T, Fig. 1A) or in the room with full-spectrum normal light (room N, Fig. 1B). In room T, melatonin concentrations were significantly increased at B2 as compared to $\mathrm{B} 1$ on the $2^{\text {nd }}$ and $3^{\text {rd }}$ night of the study, while in room $\mathrm{N}$ a significant change could only be observed at night 2 . To gain further insight into the potentially distinct effects of the different illuminations on melatonin synthesis, the differences in its serum concentrations between B2 and B1 were calculated and compared between the two lighting conditions. $\Delta \mathrm{B} 2 \mathrm{~B} 1$ values were significantly lower in room $\mathrm{N}$ at night 1 and 2 of the study when compared to the respective data obtained in room $\mathrm{T}$. This stabilizing effect of filtered light on melatonin generation was confirmed by direct comparison of serum melatonin concentrations obtained from samples in room $\mathrm{T}$ and room $\mathrm{N}$, respectively. While no differences in melatonin levels between both office accomodations could be found in specimen collected at the beginning of each night of the study, melatonin was significantly lower in room $\mathrm{N}$ at B2 when compared to the corresponding values in room $\mathrm{T}$ at every night of the experiments.

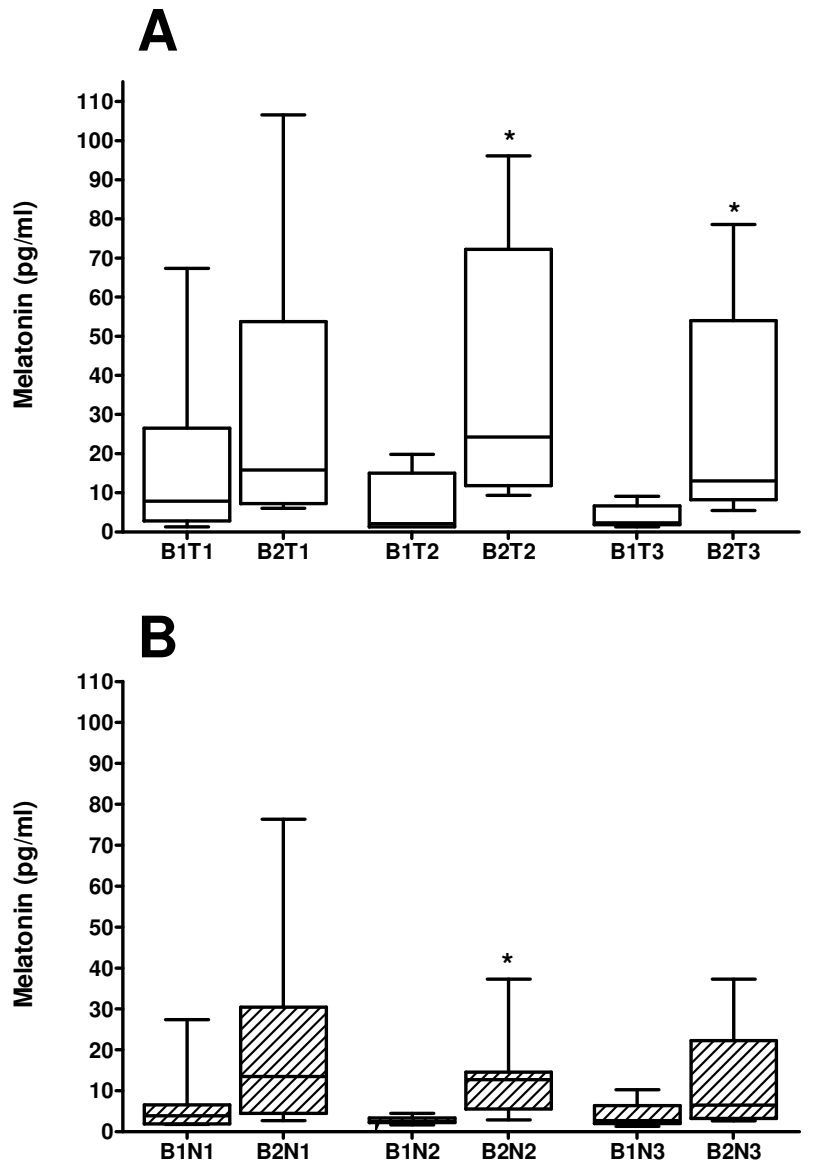

Fig. (1). Serum melatonin concentrations on the first, second, and third night either in a lighting environment with reduced short wavelength components (T1, T2, and T3; Fig. A) or in an unfiltered bright light environment (N1, N2, and N3; Fig. B). Time points of specimen collection were 10:00 p.m. (B1), and 06:00 a.m. (B2). Data are given as median together with the $25^{\text {th }}$ and the $75^{\text {th }}$ quartile, whiskers represent highest and lowest values.

$* \mathrm{P}<0.05$ as compared to time point $\mathrm{B} 1$ of the respective night of the study.

With respect to red blood count, no significant differences between B2 and B1 could be found under both lighting conditions at any night of the study (Table 1). Erythrocyte count as well as hemoglobin concentrations and hematocrit remained unchanged in the course of the study. It should be noted that in all study participants the corresponding values were within the normal range. Regarding white blood count, we observed significant increases in the percentage of lymphocytes and eosinophils when comparing B2 with B1 at every night of the study that were independent of the illumi- 
Table 1. Results of Red and White Blood Count. Data are Given as Mean \pm SEM

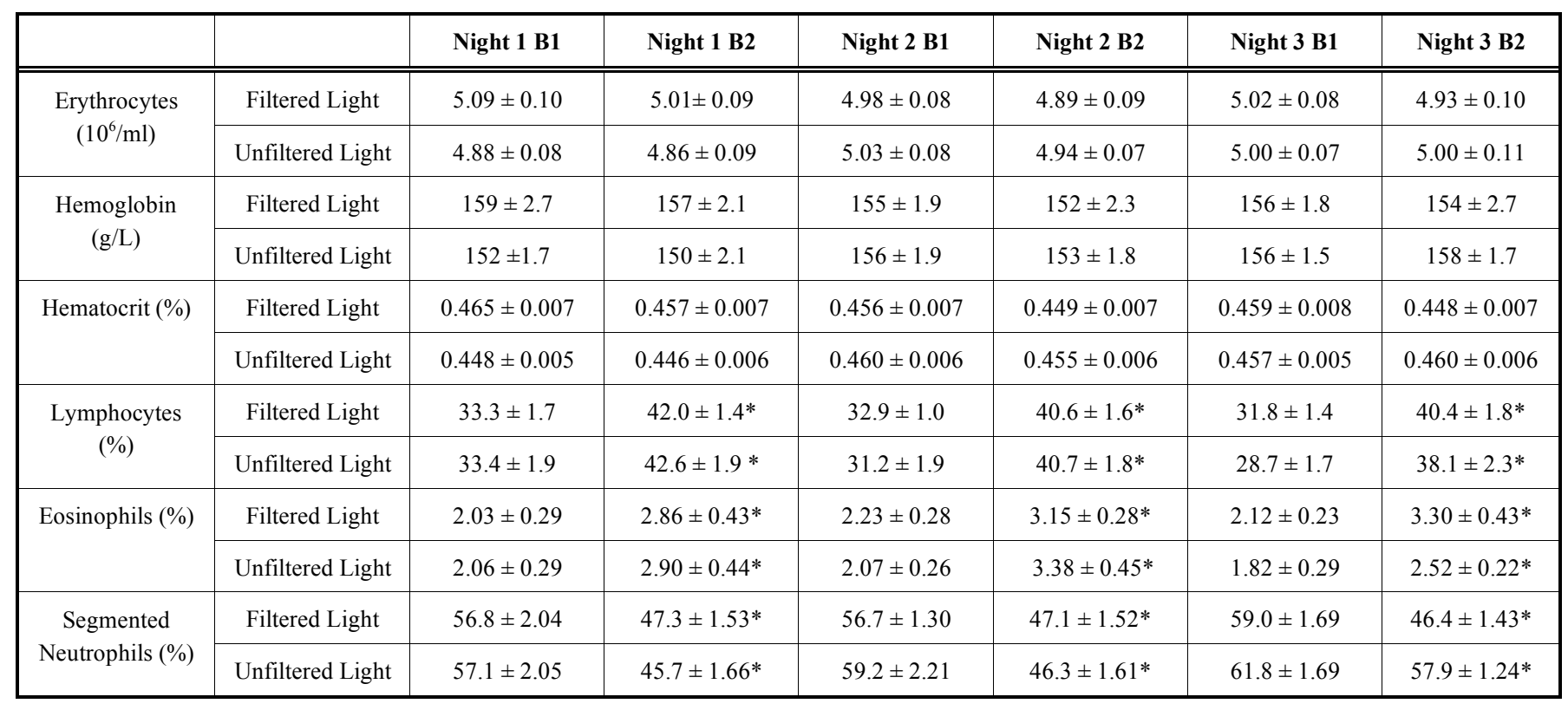

B $(1,2)$ : The first or second time point of blood sample collection during the experimental night shift $(1=10: 00$ p.m.; $2=06: 00$ a.m. $)$.

$* \mathrm{P}<0.05$ as compared to the first measurement (B1) on the respective night of the study protocol (Wilcoxon matched pairs test).

nation. In contrast, percentage of segmented neutrophils revealed significant depletions in the course of each night of the experiments in both filtered and full-spectrum office environments (Table 1). Again, $\triangle \mathrm{B} 2 \mathrm{~B} 1$ values were calculated for each parameter and for every night of the study. Statistical comparison of these differences between room $\mathrm{T}$ and room $\mathrm{N}$ did not point out a discriminative effect of the lighting condition on red or white blood count.

Data of resting systolic and diastolic blood pressure are summarized in Figs. (2 and 3), respectively and revealed a significant overall time effect (evaluated by Friedman test for matched groups with repeated measurements) in room $\mathrm{T}$ on the first night ( $p=0.0241$ for systolic pressure (Fig. 2A) and $p=0.0140$ for diastolic pressure (Fig. 3A)) and on the third night $(\mathrm{p}=0.0092$ for systolic pressure (Fig. 2B) and $\mathrm{p}=$ 0.0209 for diastolic pressure (Fig. 3B)) of the experiments. In contrast, no significant changes were observed in the office with full-spectrum light. Friedman analyses of heart rate values (Fig. 4) resulted in significant overall time effects in room $\mathrm{T}$ at night $1(\mathrm{p}=0.0054$, Fig. 4A), night $2(\mathrm{p}=0.0230$, Fig. 4B), and night $3(\mathrm{p}=0.0020$, Fig. 4C) of the study, while in room $\mathrm{N}$ the respective data were significant at night $2(p=0.0062$, Fig. 4E) and night $3(p=0.0139$, Fig. 4F).

Results of the main scoring parameters of the ability testings are summarized in Table 2. To mark the level of activation, fusion (FUF) and flicker (FLF) frequency were determined at the beginning (A1) and at the end (A2) of every night of the study. While FUF remained unchanged throughout the experiments in room $\mathrm{T}$ and room $\mathrm{N}$, FLF turned out to be significantly reduced under both filtered and full-spectrum lighting conditions at the end of the night. Continuous attention tests were performed twice per night to assess the influence of the different lighting environments on long-term selective concentration ability of the study participants. The main parameter for analyses was the sum of correct hits (SUMC). SUMC was significantly lower at A2 as compared to A1 at night 2 in room T and at both nights 1 and 2 in room N. Regarding the auxiliary parameters of continuous attention, MEANC was increased at A2 on the second night in filtered light and at nights 1 and 3 under bright light conditions. SUMI was significantly augmented at A2 as compared to $\mathrm{A} 1$ at night 2 in room $\mathrm{T}$ and at every night of the study in room N, while MEANI was significantly higher at A2 in both rooms on the second night when compared to the initial values obtained at A1. Cognitive speed (processing, perception, and motor response organization for choice reaction tasks) were determined via Reaction Time Analyses at 11:30 p.m. (A1) and at 3:00 a.m. (A2). Taken together, the main scoring parameters IP, PER, MRO as well as CRT remained unchanged throughout the experiments and were independent of the illumination. Concerning VSRT, we observed a significant decrease at A2 on the third night in the room with unfiltered bright light when comparing to the respective data at A1. Vigilance tests were scheduled at midnight (A1) and at 3:30 a.m. (A2) in order to evaluate attention of study participants under continuous stress. When compared to A1 values, the major parameter NUMC turned out to be significantly reduced at the end of night 1 and 2 in room $\mathrm{T}$, but only at night 1 in room $\mathrm{N}$. With respect to auxiliary parameters, MRTC was significantly higher in both rooms at A2 on the first night of the study, and NUMI was increased at $\mathrm{A} 2$ as compared to $\mathrm{A} 1$ at night 2 in room $\mathrm{N}$.

\section{DISCUSSION}

Melatonin is one of the best studied circadian parameters in humans. The $24 \mathrm{~h}$-profile of melatonin shows a peak at approximately 4:00 a.m. Since melatonin production declines with age, we have chosen to enroll volunteers belonging to an age group that still has a high melatonin production capability as well as a low inter-individual variability in melatonin synthesis [17]. In our study, serum melatonin concentrations were highest at the second time point of blood 

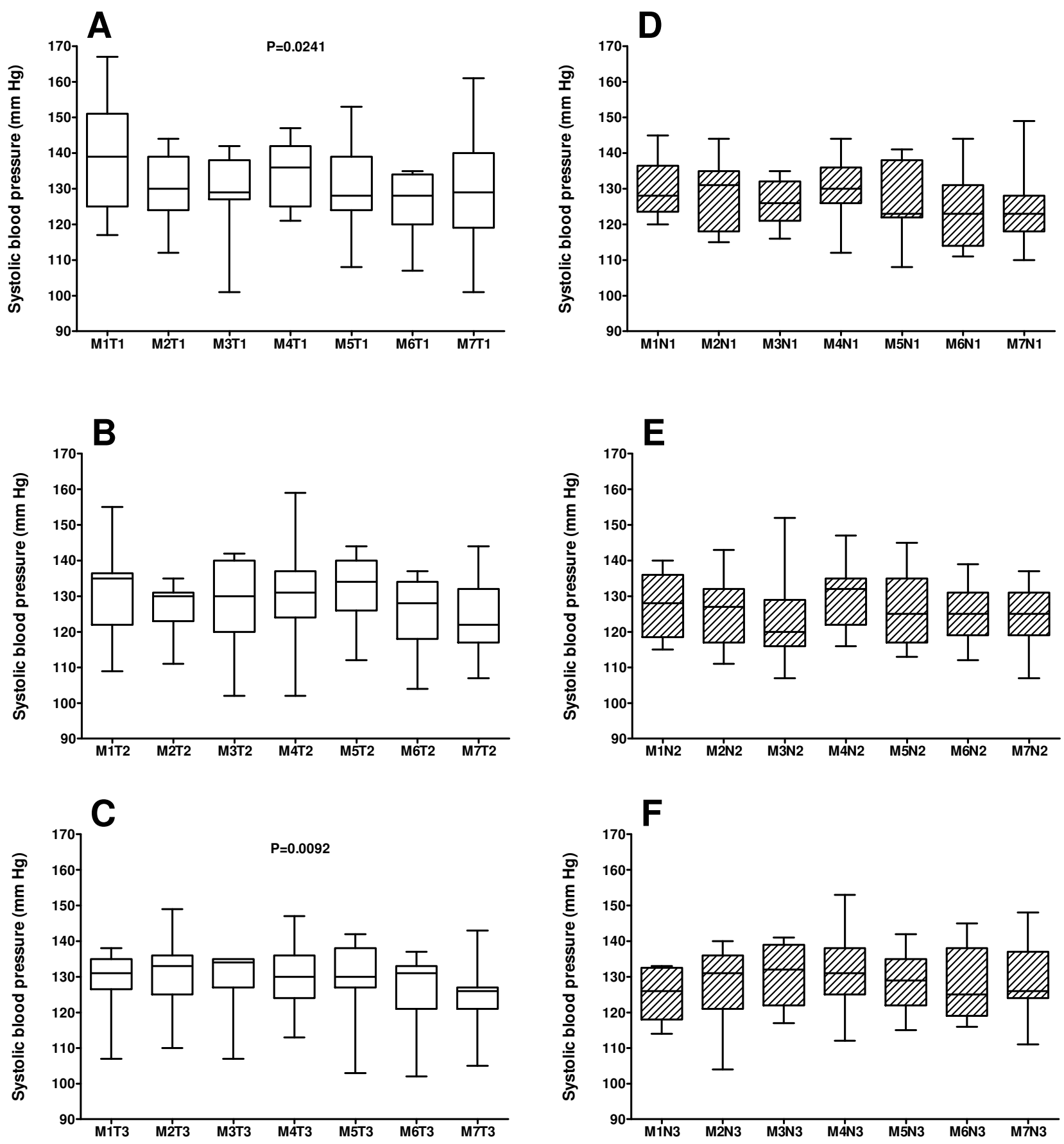

Fig. (2). Time course of systolic blood pressure on the first, second, and third night either in a lighting environment with reduced short wavelength components (T1, T2, and T3; Figs. A, B, and C, respectively) or in an unfiltered bright light environment (N1, N2, and N3; Figs. D, E, and F, respectively). Time points for each measurement were: M1 at 10:30 p.m.; M2 at 11:30 p.m.; M3 at 0:15 a.m.; M4 at 1:45 a.m.; M5 at 2:45 a.m.; M6 at 3:30 a.m.; and M7 at 5:30 a.m. Data are given as median together with the $25^{\text {th }}$ and the $75^{\text {th }}$ quartile, whiskers represent highest and lowest values. Friedman tests for matched groups with repeated measures were performed to analyze potential time effects, resulting P-values are given in the corresponding panel.

sample collection, i.e. at 6:00 a.m., which is in congruence with the reported circadian rhythm of the hormone. With respect to the different illuminations, significantly lower melatonin values in unfiltered light as compared to light with reduced short wavelength components found in our study confirms previous observations establishing bright light as a potent suppressor of pinealocyte melatonin synthesis. Thus, altered diurnal melatonin profiles are a regular finding among shift workers $[18,19]$, albeit there seems to be a ca- pacity to adapt to a pattern of shift work with a higher number of consecutive nights and a fixed sleep schedule during the days [20]. Comparable to the preserving effect of filtered light on melatonin generation observed in our experiments, Kayumov et al. [21] reported a normal night time profile of melatonin in a simulated shift work model in participants wearing light-filtering goggles that blocked wavelengths of less than $530 \mathrm{~nm}$. With respect to the key parameter melatonin, the present study provides evidence that the benefits of 

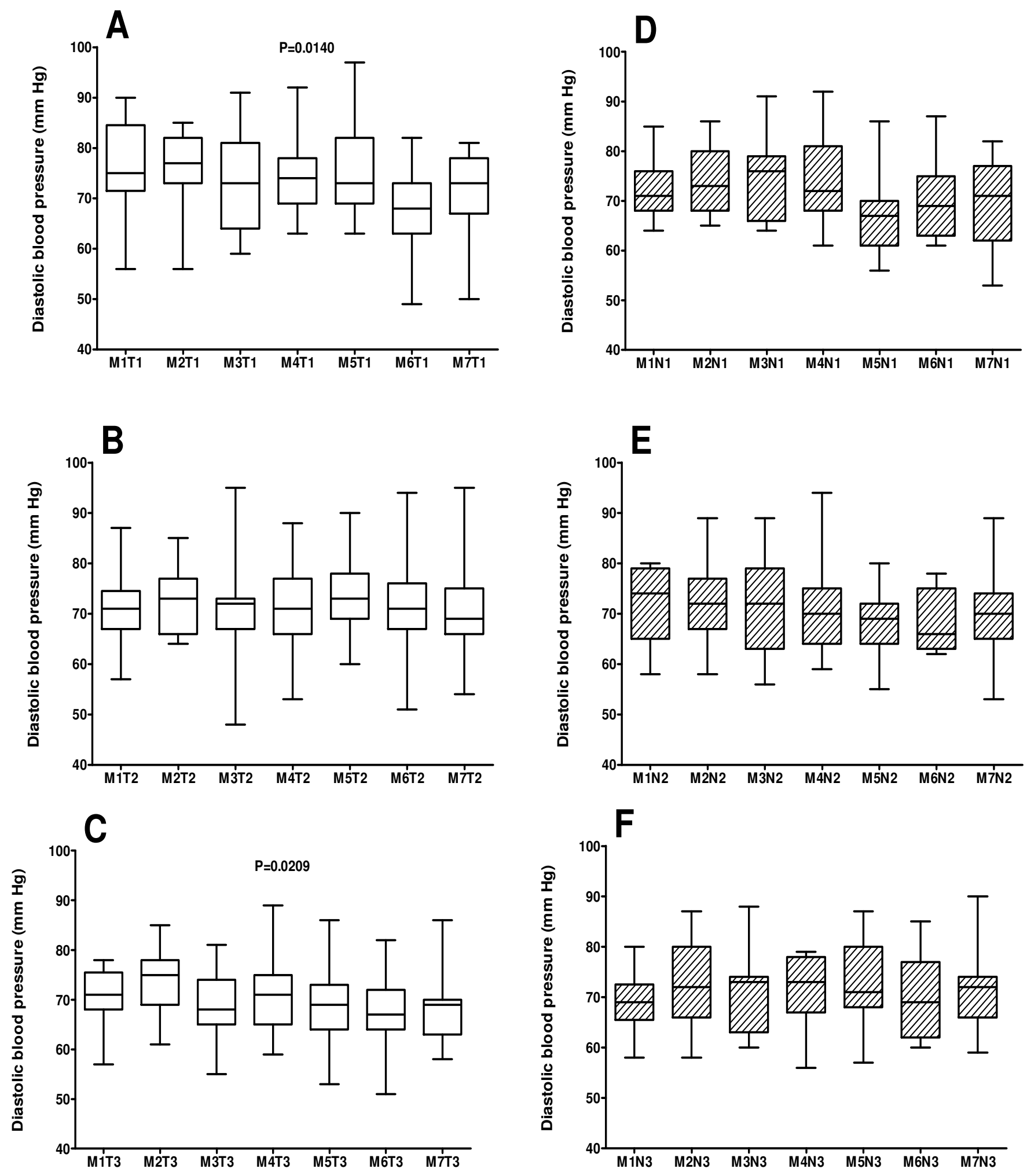

Fig. (3). Time course of diastolic blood pressure on the first, second, and third night either in a lighting environment with reduced short wavelength components (T1, T2, and T3; Figs. A, B, and C, respectively) or in an unfiltered bright light environment (N1, N2, and N3; Figs. D, E, and $\mathbf{F}$, respectively). Time points for each measurement were: M1 at 10:30 p.m.; M2 at 11:30 p.m.; M3 at 0:15 a.m.; M4 at 1:45 a.m.; M5 at 2:45 a.m.; M6 at 3:30 a.m.; and M7 at 5:30 a.m. Data are given as median together with the $25^{\text {th }}$ and the $75^{\text {th }}$ quartile, whiskers represent highest and lowest values. Friedman tests for matched groups with repeated measures were performed to analyze potential time effects, resulting P-values are given in the corresponding panel. 

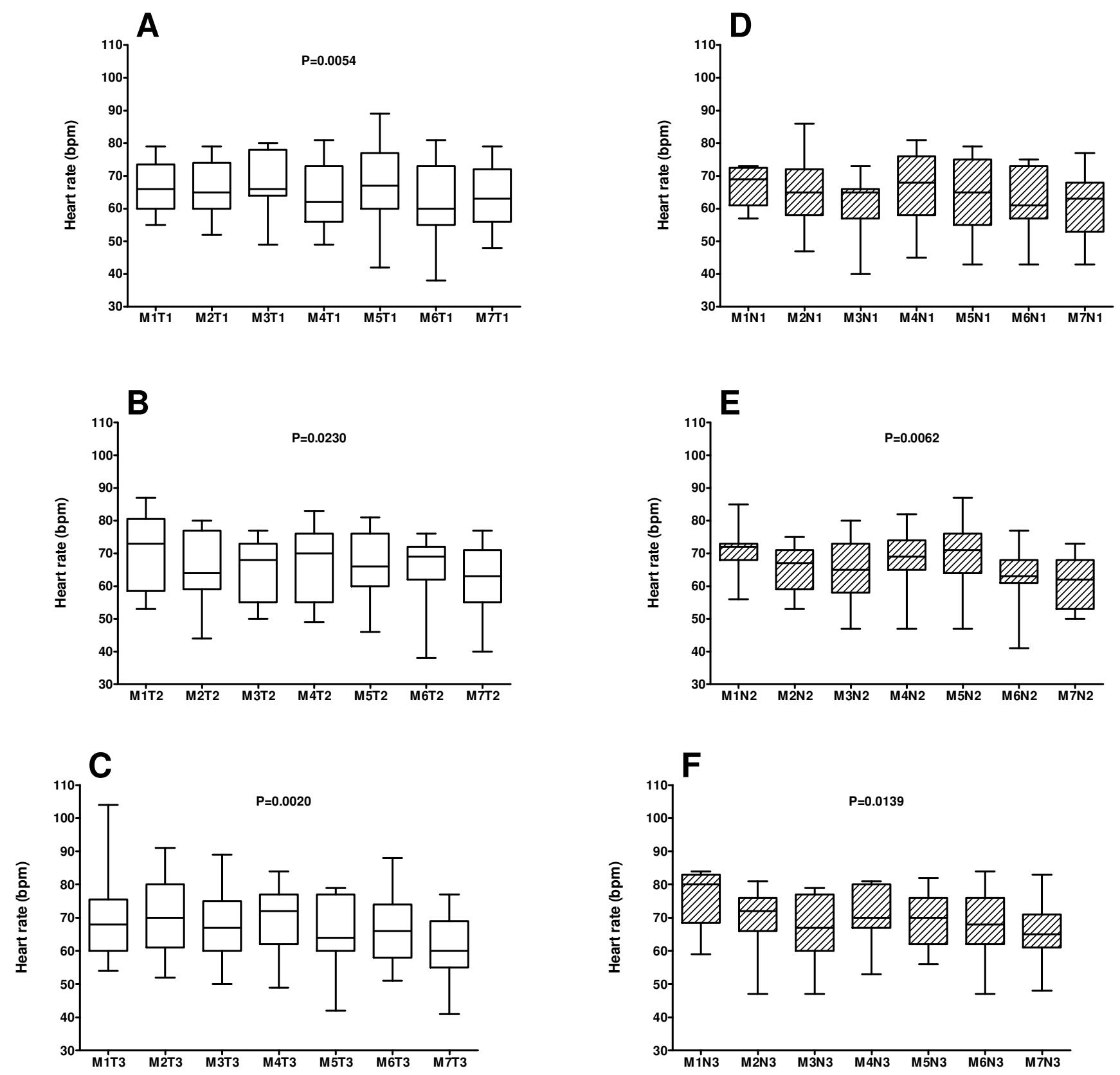

Fig. (4). Time course of heart rate on the first, second, and third night either in a lighting environment with reduced short wavelength components (T1, T2, and T3; Figs. A, B, and C, respectively) or in an unfiltered bright light environment (N1, N2, and N3; Figs. D, E, and F, respectively). Time points for each measurement were: M1 at 10:30 p.m.; M2 at 11:30 p.m.; M3 at 0:15 a.m.; M4 at 1:45 a.m.; M5 at 2:45 a.m.; M6 at 3:30 a.m.; and M7 at 5:30 a.m. Data are given as median together with the $25^{\text {th }}$ and the $75^{\text {th }}$ quartile, whiskers represent highest and lowest values. Friedman tests for matched groups with repeated measures were performed to analyze potential time effects, resulting P-values are given in the corresponding panel.

reducing short-wavelength light can be achieved in an almost natural office accomodation, too.

Erythrocyte count, hematocrit, and hemoglobin concentration remained unchanged throughout the experiments and were not influenced by the different lighting environments. An increase in hematocrit and hemoglobin concentrations with average morning values being significantly higher than the respective data of the evening before have been reported in the literature $[22,23]$. Since these changes were due to nocturnal reductions in plasma volume, we conclude that our study participants kept fluid loss and fluid replacement in balance in the course of the study. Nocturnal increases in eosinophils and lymphocytes as well as decreases in segmented neutrophils observed in our study might be due to circadian variations in glucocorticosteroid synthesis, predominantely cortisol. The physiological 24 h-profile of cortisol establishes minimal serum values in the evening and a surge in the early morning hours. Glucocorticosteroids have been considered to regulate immune cell systems through modulation of apoptotic cell death. Thus, experimentally 
Table 2. Results of Ability Test Systems (Vienna Test System ${ }^{\circledR}$, Dr. G. Schuhfried Ltd., Moedling, Austria). Data are Given as Mean \pm SEM

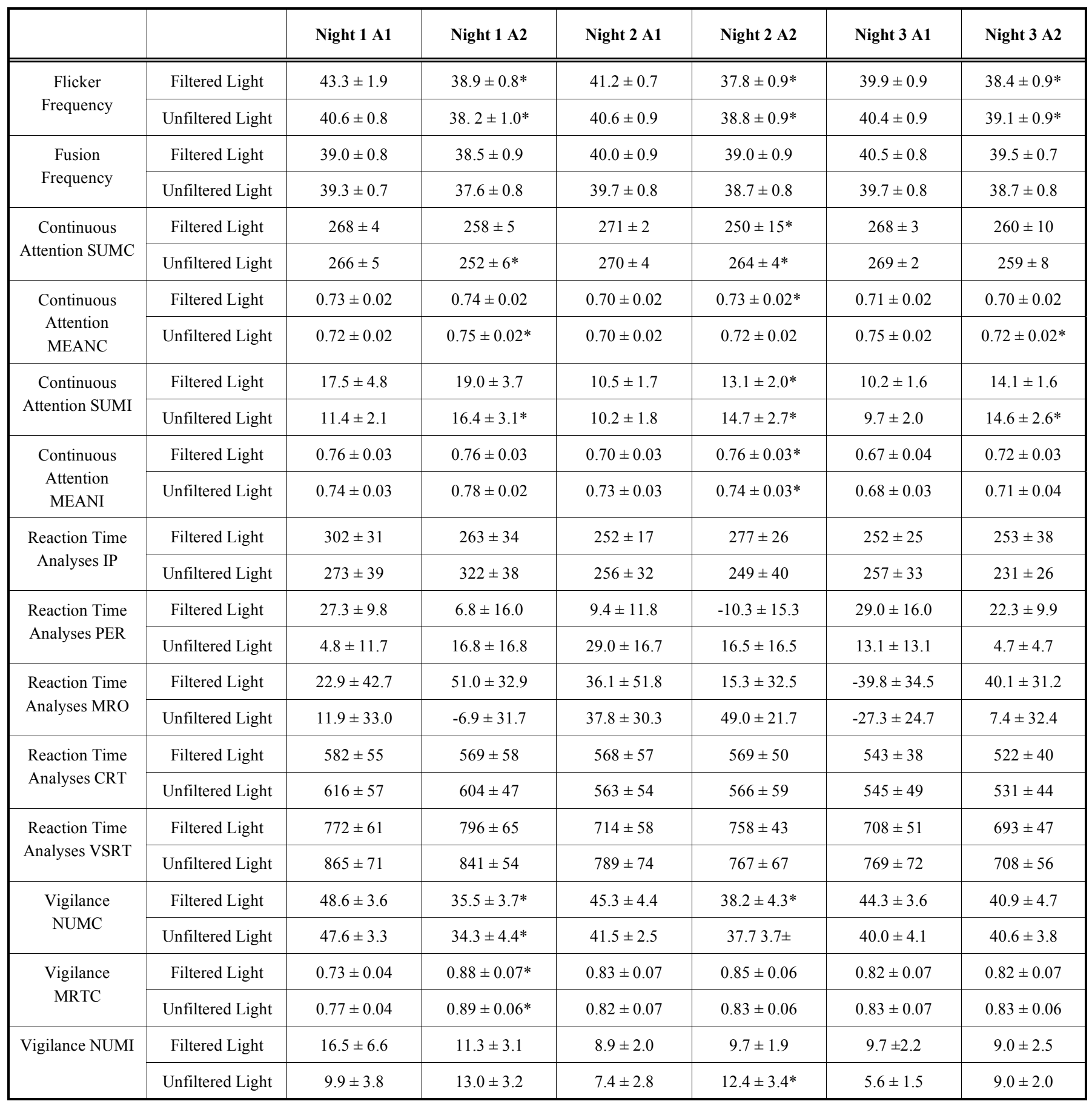

A $(1,2)$ : the first or second ability test during the experimental night shift; specific time points for the start of the respective tests were: Flicker/Fusion frequency A1 $=10: 15$ p.m., Flicker/Fusion Frequency A2 = 5:30 a.m.; Continuous Attention A1 = 11:00 p.m., Continuous Attention A2 = 2:15 a.m.; Reaction Time Analysis A1 = 11:35 p.m., Reaction Time Analysis A2 = 3:00 a.m.; Vigilance A1 = midnight, Vigilance A2: 3:30 a.m.

$* \mathrm{P}<0.05$ as compared to the first measurement (A1) on the respective night of the study protocol (Wilcoxon matched pairs test).

Abbreviations: $\mathrm{CRT}=$ choice reaction time; $\mathrm{IP}=$ information processing; MEANC = mean time for correct hits; MEANI $=$ mean time for incorrect hits; $\mathrm{MRO}=$ motor response organization; MRTC $=$ mean value of reaction time for correct hits; NUMC $=$ number of correct hits; NUMI = number of incorrect hits; PER = perception; $\mathrm{SUMC}=$ sum of correct hits; SUMI = sum of incorrect hits; VSRT = reaction time for visual search

applied dexamethasone, prednisolone, or hydrocortisone induced apoptosis in eosinophils and lymphocytes, while programmed cell death in neutrophils was averted [24,25]. Since we did not measure serum cortisol levels, we can only speculate that a nocturnal decrease in glucocorticosteroid synthesis and release is responsible for the alterations in white blood count. Interestingly, variations in white blood count have recently been documented by Nishitani and Sakakibara [26] to be more pronounced in shift workers as compared to daytime workers. The authors suggested that quality of sleep might affect white blood status. It should be noted that the changes did not differ between the room with 
filtered light and the room with full-spectrum light, thus being independent of the chosen illumination.

Blood pressure follows a distinct circadian profile with a characteristic decline during sleep (nocturnal values being $10-20 \%$ lower than the mean daytime measurements) followed by a surge in the early morning hours [27]. Since circadian rhythm in blood pressure is largely dependent on regular sleep-wake patterns, night shift is usually associated with rapid alterations in the course of $24 \mathrm{~h}$ systolic and diastolic blood pressure behaviour [28]. In our study, systolic and diastolic blood pressure remained within a normal circadian design in the room with filtered light at night 1 and 3 of the study, while there were no significant overall time effects in the room with full-spectrum light, implying that the circadian profile was deranged. However, the preserving effect of light with reduced short wavelength components on nocturnal variations in blood pressure values was not consistent throughout the experiments since it was not present on the second night. Circadian oscillations of the autonomic nervous system with a prevalence of sympathetic tone at daytime and a switch towards parasympathetic predominance at night results in a nocturnal decrease in heart rate $[29,30]$. Although the effects of an irregular sleep-wake cycle on heart rate associated with shift work are discussed controversially [31,32], light with intensities sufficient to affect circadian patterns of melatonin has been shown to influence heart rate in humans [33]. Friedman analyses of our data yielded a significant change in nocturnal heart rate with decreases in the course of all nights in filtered light and at both nights 2 and 3 in full-spectrum light. These data do not support the concept of a differentiating effect of the chosen lighting environments on heart rate values.

Maintenance of a physiological circadian rhythm represents only one ergonomical aspect for the design of workplace illuminations. Another important topic is the employees' efficiency and well-being. In this regard, pulses of bright light have been reported to exert beneficial effects on alertness as well as performance in night shift workers [34,35]. In a study by Eastman and co-workers [36], high intensities of bright light (5000 lux) modulated phase shifting of temperature which was associated with better daytime recovery, fatigue, and overall mood as compared to 500 lux dim light controls in a simulated night shift model. These data demonstrate a favourable effect of intermittent nocturnal exposure to bright light. Therefore, we had to take into account a potentially detrimental impact of light with reduced short wavelength components on productivity and performance of our study participants. In order to assess this question, various ability tests were performed throughout the course of the study. Flicker/Fusion Frequency analysis is regarded as an indicator for central-nervous activation. In our study, flicker frequency was significantly decreased at the end of each night shift, which can be interpreted as a reduction in volunteer's arousal. However, these changes were observed under both lighting conditions and statistical comparison of $\triangle \mathrm{A} 2 \mathrm{~A} 1$ values did not result in a significantly different impact of workplace illumination on this parameter. Continuous Attention testing is designed to evaluate longterm attention and concentration ability, and thereby allowing an indirect assessment of general performance. Although some of the parameters included in the respective analyses were significantly altered when comparing the second with the first time point of data collection, we could not detect an unambiguous deleterious effect of filtered light on the level of attentiveness of our study participants. As with Flicker/Fusion Frequency, $\Delta \mathrm{A} 2 \mathrm{~A} 1$ values could not discriminate between filtered and full-spectrum light. According to the different stages of activity regulation, we could not observe significant alterations in information processing, perception, and motor response organization measured by Reaction Time Analyses on the three nights of the study. These results do not support the hypothesis that filtered light deteriorates cognitive speed in our experimental night shift model. However, there was a significant drop in the number of correct hits assessed during Vigilance testing at both night 1 and 2 under filtered light that was present only at night 1 under full-spectrum light. Although $\triangle \mathrm{A} 2 \mathrm{~A} 1$ values for this parameter did not differ between the two office accomodations at any night of the study, our findings indicate a possible impairment of the performance of monotonous monitoring tasks in our test light system with reduced short wavelength components. Apart from the Vigilance test data, filtered light did not affect alertness and performance in our experimental setup. Thus, our data do not support the concept of a detrimental effect of maintaining circadian melatonin rhythm on fatigue, precision, or on-the-job attentiveness. This view is confirmed by Paul et al. [37], who did not find impaired psychomotor performance (attentiveness, reaction time, motor response organization) in volunteers treated with exogenously applied melatonin. Moreover, in the above-mentioned study by Kayumov and co-workers [21], preservation of nocturnal melatonin levels did not exert an influence on performance data or subjective sleepiness as well.

\section{CONCLUSION}

The aim of this study was to investigate the effects of a lighting environment with reduced short-wavelength components on physiological parameters following a circadian pattern as well as on parameters of performance and efficiency in a simulated night shift setup. The major finding of our study is the maintenance of the nocturnal rhythm of melatonin under test light conditions as compared to unfiltered bright light which was not accompanied by losses in the volunteer's alertness or general performance. With respect to the potentially harmful effects of disturbing a regular circadian rhythm, installation of workplaces with adapted illumination may provide a benefit for employees working night shifts.

\section{ACKNOWLEDGEMENTS}

The authors would like to thank all volunteers and the local team from Bartenbach LichtLabor, Aldrans, Austria as well as the rest of the staff involved in the study. We gratefully acknowledge the expert technical assistance of Drs. G. Falkensammer and A. Hammerer-Lercher at the Central Institute for Medical and Chemical Laboratory Diagnostics, University Hospital Innsbruck-TILAK, Innsbruck, Austria. Statistical analyses were performed by Prof. Dr. A. Klingler at the Theoretical Surgery Unit of the Department of General and Transplant Surgery, Medical University Innsbruck, Austria. This study was financially supported by the Lighting Network "K-Licht", Aldrans, Austria, and in part by the TI- 
LAK GesmbH (Tiroler Landeskrankenanstalten), Innsbruck, Austria as well as by means of the "Bundesministerium fuer Wirtschaft und Arbeit", Austria.

\section{REFERENCES}

[1] Akerstedt T. Sleepiness as a consequence of shift work. Sleep 1988; 11: 17-34

[2] Cavallo A, Jaskiewicz J, Ris MD. Impact of night-float rotation on sleep, mood, and alertness: the resident's perception. Chronobiol Int 2002; 19: 893-902.

[3] Kawachi I, Colditz GA, Stampfer MJ, et al. Prospective study of shift work and risk of coronary heart disease in women. Circulation 1995; 92: 3178-82.

[4] Scott AJ, Monk TH, Brink LL. Shiftwork as a Risk Factor for Depression: A Pilot Study. Int J Occup Environ Health 1997; 3: S2S9.

[5] Hansen J. Light at night, shiftwork, and breast cancer risk. J Natl Cancer Inst 2001; 93: 1513-5.

[6] Schernhammer ES, Laden F, Speizer FE, et al. Night-shift work and risk of colorectal cancer in the nurses' health study. J Natl Cancer Inst 2003; 95: 825-8.

[7] Schwartzbaum J, Ahlbom A, Feychting M. Cohort study of cancer risk among male and female shift workers. Scand J Work Environ Health 2007; 33: 336-43.

[8] Berson DM, Dunn FA, Takao M. Phototransduction by retinal ganglion cells that set the circadian clock. Science 2002; 295: 1070-3.

[9] Dacey DM, Liao HW, Peterson BB, et al. Melanopsin-expressing ganglion cells in primate retina signal colour and irradiance and project to the LGN. Nature 2005; 433: 749-54.

[10] Provencio I, Rodriguez IR, Jiang G, Hayes WP, Moreira EF, Rollag MD. A novel human opsin in the inner retina. J Neurosci 2000; 20: $600-5$.

[11] Weitzman ED, Weinberg U, D'Eletto R, et al. Studies of the 24 hour rhythm of melatonin in man. J Neural Transm Suppl 1978; 325-37.

[12] Klein DC, Weller JL. Rapid light-induced decrease in pineal serotonin N-acetyltransferase activity. Science 1972; 177: 532-3.

[13] Lewy AJ, Wehr TA, Goodwin FK, Newsome DA, Markey SP. Light suppresses melatonin secretion in humans. Science 1980; 210: 1267-9.

[14] Zeitzer JM, Dijk DJ, Kronauer R, Brown E, Czeisler C. Sensitivity of the human circadian pacemaker to nocturnal light: melatonin phase resetting and suppression. J Physiol 2000; 526 Pt 3: 695-702.

[15] Hattar S, Liao HW, Takao M, Berson DM, Yau KW. Melanopsincontaining retinal ganglion cells: architecture, projections, and intrinsic photosensitivity. Science 2002; 295: 1065-70.

[16] Lockley SW, Brainard GC, Czeisler CA. High sensitivity of the human circadian melatonin rhythm to resetting by short wavelength light. J Clin Endocrinol Metab 2003; 88: 4502-5.

[17] Wetterberg L, Bergiannaki JD, Paparrigopoulos T, von Knorring L, Eberhard G, Bratlid T, Yuwiler A. Normative melatonin excretion: a multinational study. Psychoneuroendocrinology 1999; 4: 209-26.

[18] Borugian MJ, Gallagher RP, Friesen MC, Switzer TF, Aronson KJ. Twenty-four-hour light exposure and melatonin levels among shift workers. J Occup Environ Med 2005; 47: 1268-75.
[19] Yamauchi H. Effects of night work on urinary excretion rates of 6sulfatoxymelatonin, norepinephrine and estriol in pregnant women. Ind Health 2004; 42: 268-76.

[20] Roach GD, Lamond N, Dorrian J, et al. Changes in the concentration of urinary 6-sulphatoxymelatonin during a week of simulated night work. Ind Health 2005; 43: 193-6.

[21] Kayumov L, Casper RF, Hawa RJ, et al. Blocking low-wavelength light prevents nocturnal melatonin suppression with no adverse effect on performance during simulated shift work. J Clin Endocrinol Metab 2005; 90: 2755-61.

[22] Jones AR, Twedt D, Swaim W, Gottfried E. Diurnal change of blood count analytes in normal subjects. Am J Clin Pathol 1996; 106: 723-7.

[23] Talan MI, Engel BT, Kawate R. Overnight increases in haematocrit: additional evidence for a nocturnal fall in plasma volume. Acta Physiol Scand 1992; 144: 473-6.

[24] Lill-Elghanian D, Schwartz K, King L, Fraker P. Glucocorticoidinduced apoptosis in early B cells from human bone marrow. Exp Biol Med (Maywood ) 2002; 227: 763-70.

[25] Meagher LC, Cousin JM, Seck1 JR, Haslett C. Opposing effects of glucocorticoids on the rate of apoptosis in neutrophilic and eosinophilic granulocytes. J Immunol 1996; 156: 4422-8.

[26] Nishitani N, Sakakibara H. Subjective poor sleep and white blood cell count in male Japanese workers. Ind Health 2007; 45: 296-300.

[27] Millar-Craig MW, Bishop CN, Raftery EB. Circadian variation of blood-pressure. Lancet 1978; 1: 795-7.

[28] Sundberg S, Kohvakka A, Gordin A. Rapid reversal of circadian blood pressure rhythm in shift workers. J Hypertens 1988; 6: 393-6.

[29] Hayano J, Sakakibara Y, Yamada M, et al. Diurnal variations in vagal and sympathetic cardiac control. Am J Physiol 1990; 258: H642-6.

[30] Somers VK, Dyken ME, Mark AL, Abboud FM. Sympatheticnerve activity during sleep in normal subjects. N Engl J Med 1993; 328: 303-7.

[31] Burgess HJ, Trinder J, Kim Y, Luke D. Sleep and circadian influences on cardiac autonomic nervous system activity. Am J Physiol 1997; 273: H1761-8.

[32] Carrington M, Walsh M, Stambas T, Kleiman J, Trinder J. The influence of sleep onset on the diurnal variation in cardiac activity and cardiac control. J Sleep Res 2003; 12: 213-21.

[33] Scheer FA, Van Doornen LJ, Buijs RM. Light and diurnal cycle affect autonomic cardiac balance in human; possible role for the biological clock. Auton Neurosci 2004; 110: 44-8.

[34] Dawson D, Campbell SS. Timed exposure to bright light improves sleep and alertness during simulated night shifts. Sleep 1991; 14: 511-6.

[35] Foret J, Daurat A, Tirilly G. Effect of bright light at night on core temperature, subjective alertness and performance as a function of exposure time. Scand J Work Environ Health 1998; 24(Suppl 3): 115-20.

[36] Eastman CI, Liu L, Fogg LF. Circadian rhythm adaptation to simulated night shift work: effect of nocturnal bright-light duration. Sleep 1995; 18: 399-407.

[37] Paul MA, Gray G, Kenny G, Pigeau RA. Impact of melatonin, zaleplon, zopiclone, and temazepam on psychomotor performance. Aviat Space Environ Med 2003; 74: 1263-70.

(C) Hoffmann et al.; Licensee Bentham Open.

This is an open access article licensed under the terms of the Creative Commons Attribution Non-Commercial License (http://creativecommons.org/licenses/by-nc/3.0/) which permits unrestricted, non-commercial use, distribution and reproduction in any medium, provided the work is properly cited. 